APPENDIX V

附錄五

\title{
Donors to the Li Fang-Kuei Society Endowment Fund 紀念李方桂先生中國語言學研究學會贊助人名單
}

\author{
Hiroyuki Akitani 秋谷裕幸 \\ Anonymous 無名氏 \\ Navavan Bandhumedha \\ Chin-Chuan Cheng 鄭錦全 \\ Hung-Nin Samuel and Adaline Cheung 張洪年伉儷 \\ Dusdeporn Chumnirokasant \\ Mr. \& Mrs. South Coblin 柯蔚南伉麗 \\ Kanchanee Dulalumpa \\ East Bay Community Foundation \\ Hwang-Cherng \& Cai Chan Gong 龔煌城、蔡盛 \\ Zev Handel \& Ju Namkung \\ Kay Hashimoto 橋本京 \\ Ho Dah-An 何大安 \\ C.-T. James \& Emily Huang 黃正德伉儷 \\ Cheng Yueh-Mei Lee \\ Annie Li 李安德 \\ Li Family Education Fund \\ Jen-Kuei Li 李王癸 \\ Peter \& Marjorie Li 李培德伉儷 \\ Chinfa Lien 連金發 \\ Ying Liu 劉穎 \\ Direk Lawansiri \\ Ajchara Mahaphirom \\ Prapin Manomaivibool 巴平 \\ Lindy Li Mark 李林德 \\ Tsu-Lin Mei 梅祖麟 \\ Alexis Michaud 米克 \\ Weera Ostapirat 許家平 \\ Alain Peyraube 貝羅貝 \\ Vichin Panupong \\ Sasiporn Petcharapirat \\ Nisa Sakdejyont \\ Kanlayanee Sitasuwan \\ Jingtao Sun 孫景濤
}


Ken-ichi Takashima 高嶋謙一

Pang-Hsin and Chen Chi Ting 丁邦新、陳琪

Surangsri Tonsiengsom

William S.-Y. Wang 王士元

Carine Yuk-man Yiu 姚玉敏

Anne Yue-Hashimoto 余霭芹

Xiaonong Zhu 朱曉農 